\title{
Effects of subgrid-scale modeling on time correlations in large eddy simulation
}

\author{
Guo-Wei He ${ }^{\text {a) }}$ \\ ICASE, Mail Stop 132C, NASA Langley Research Center, Hampton, Virginia 23681-2199 \\ and LNM, Institute of Mechanics, Chinese Academy of Sciences, Beijing 100080, China \\ R. Rubinstein \\ Mail Stop 128, NASA Langley Research Center, Hampton, Virginia 23681-2199 \\ Lian-Ping Wang \\ Department of Mechanical Engineering, 126 Spencer Laboratory, University of Delaware, Newark, \\ Delaware 19716
}

(Received 5 September 2001; accepted 17 April 2002; published 24 May 2002)

\begin{abstract}
The effects of the unresolved subgrid-scale (SGS) motions on the energy balance of the resolved scales in large eddy simulation (LES) have been investigated actively because modeling the energy transfer between the resolved and unresolved scales is crucial to constructing accurate SGS models. But the subgrid scales not only modify the energy balance, they also contribute to temporal decorrelation of the resolved scales. The importance of this effect in applications including the predictability problem and the evaluation of sound radiation by turbulent flows motivates the present study of the effect of SGS modeling on turbulent time correlations. This paper compares the two-point, two-time Eulerian velocity correlation in isotropic homogeneous turbulence evaluated by direct numerical simulation (DNS) with the correlations evaluated by LES using a standard spectral eddy viscosity. It proves convenient to express the two-point correlations in terms of spatial Fourier decomposition of the velocity field. The LES fields are more coherent than the DNS fields: their time correlations decay more slowly at all resolved scales of motion and both their integral scales and microscales are larger than those of the DNS field. Filtering alone is not responsible for this effect: in the Fourier representation, the time correlations of the filtered DNS field are identical to those of the DNS field itself. The possibility of modeling the decorrelating effects of the unresolved scales of motion by including a random force in the model is briefly discussed. The results could have applications to the problem of computing sound sources in isotropic homogeneous turbulence by LES. (ㄷ 2002 American Institute of Physics. [DOI: 10.1063/1.1483877]
\end{abstract}

\section{INTRODUCTION}

Constructing subgrid scale (SGS) models for large eddy simulation (LES) depends on theoretical understanding of how the unresolved scales of motion influence the resolved scales. The most extensively applied class of SGS models is based on the eddy viscosity assumption: like the molecularscale thermal fluctuations, the effects of the subgrid scale motion on the resolved scales can be modeled by a viscosity; however, unlike the molecular viscosity, this eddy viscosity depends on the filter size and on certain properties of the resolved scales. The Smagorinsky model ${ }^{1}$ is the classic eddy viscosity model. Although it is no longer often applied in its original form, it is the basis of much more commonly used models like the dynamic model, ${ }^{2}$ various "mixed" models, ${ }^{3,4}$ and more specialized spectral SGS models for homogeneous turbulence. $^{5,6}$

Eddy viscosity models attempt to describe the transfer of energy between the resolved and unresolved scales. But the subgrid scales not only act as a sink and source of energy,

\footnotetext{
a) Author to whom all correspondence should be addressed; electronic mail: hgw@icase.edu
}

they also contribute, through nonlinear interactions, to the temporal decorrelation of the resolved scales. Temporal decorrelation is important in the sound radiation problem in which the turbulent time scales are directly related to the frequency of radiated sound. ${ }^{7}$ The increasing application of LES to compute sound sources ${ }^{8,9}$ and unsteady aerodynamic flows more generally, suggests that the ability of LES to predict the temporal properties of turbulence will become increasingly important. Other problems in which time correlation properties have an important role are particle dispersion and the predictability problem. ${ }^{10,11}$

This paper compares the Eulerian time correlations of spatial Fourier amplitudes computed by direct numerical simulation (DNS) with the time correlations computed by LES in the basic problem of homogeneous isotropic turbulence. This problem permits the application of a spectral eddy viscosity. The Chollet-Lesieur model ${ }^{5}$ is used because it reproduces the energetics of the resolved scales well. We conclude that the LES fields are more correlated than the DNS fields: their time correlations decay more slowly at all resolved scales of motion and their integral scales and microscales are larger than those of the DNS field.

Filtering alone can have significant effects on many tur- 
bulence quantities; however, it is evident that filtering cannot alter the time correlations of Fourier amplitudes: if $\mathbf{u}(\mathbf{k}, t)$ denotes a Fourier mode of the DNS field, then the corresponding filtered mode is

$$
\overline{\mathbf{u}}(\mathbf{k}, t)=\mathcal{F}\left(k \mid k_{c}\right) \mathbf{u}(\mathbf{k}, t),
$$

where $\mathcal{F}\left(k \mid k_{c}\right)$ is the Fourier transform of the filter function and the cutoff wave number $k_{c}$ is proportional to the inverse filter width. Regardless of the analytical form of the transfer function $F\left(k \mid k_{c}\right)$, the normalized time correlations of the exact and filtered fields trivially satisfy

$$
\frac{\left\langle\mathbf{u}(\mathbf{k}, t) \cdot \mathbf{u}\left(-\mathbf{k}, t^{\prime}\right)\right\rangle}{\langle\mathbf{u}(\mathbf{k}, t) \cdot \mathbf{u}(-\mathbf{k}, t)\rangle}=\frac{\left\langle\overline{\mathbf{u}}(\mathbf{k}, t) \cdot \overline{\mathbf{u}}\left(-\mathbf{k}, t^{\prime}\right)\right\rangle}{\langle\overline{\mathbf{u}}(\mathbf{k}, t) \cdot \overline{\mathbf{u}}(-\mathbf{k}, t)\rangle}
$$

provided $F\left(k \mid k_{c}\right) \neq 0$. It will be shown through numerical simulations that the time correlations of DNS and LES fields can differ significantly; in view of Eq. (2), the difference cannot be the result of filtering. It is natural to attribute the difference to the decorrelating effect of nonlinear interactions between the resolved and unresolved scales. A SGS model which correctly models the energy transfer between the resolved and unresolved scales of motion may not model this decorrelating effect and may consequently overpredict the coherence of the resolved scales.

The question therefore arises of how to adapt SGS models to the requirement of predicting time correlations. Previous studies of the predictability problem ${ }^{12-14}$ have suggested including a random force in the SGS model. The possibility that a random force could be chosen so that the time correlation predictions are improved is discussed briefly.

While intuitively plausible, it should be stressed that the increased correlation of LES fields is a property of NavierStokes dynamics. In Burgers turbulence, the modes become phase-locked as shocks develop; therefore, reducing the number of modes could make the resolved velocity field less correlated than the true velocity field. This comparison calls attention to a connection between phase correlations and temporal correlation properties of nonlinear fields. Phase relations in the LES field will not be the same as those in the DNS field unless the SGS model somehow restores them. The altered phase relations may either promote temporal coherence, as we find in Navier-Stokes dynamics, or reduce it, as we suggest for Burgers turbulence.

\section{SUBGRID SCALE MODELS OF ENERGY TRANSFER}

We consider time-stationary homogeneous isotropic turbulence, for which the Fourier representation is a natural setting to study SGS models. Following Kraichnan's original analysis, ${ }^{15}$ the problem of SGS modeling is best formulated in terms of the steady-state evolution equation:

$$
2 \nu k^{2} Q(k)=F(k)+T(k)
$$

where $k=|\mathbf{k}|$ and

$$
Q(k)=\left\langle u_{i}(\mathbf{k}, t) u_{i}(-\mathbf{k}, t)\right\rangle
$$

is the single-time correlation function, $\nu$ is the kinematic viscosity, $F(k)$ is the correlation of a large-scale forcing which maintains the turbulence in a steady state, and $T(k)$ is the nonlinear transfer defined by

$$
\begin{aligned}
T(k)= & \operatorname{Im}\left[P_{i m n}(\mathbf{k}) \int d \mathbf{p} d \mathbf{q} \delta(\mathbf{k}-\mathbf{p}-\mathbf{q})\right. \\
& \left.\times\left\langle u_{m}(\mathbf{p}, t) u_{n}(\mathbf{q}, t) u_{i}(-\mathbf{k}, t)\right\rangle\right] .
\end{aligned}
$$

In Eq. (5),

$$
P_{i m n}(\mathbf{k})=k_{m} P_{i n}(\mathbf{k})+k_{n} P_{i m}(\mathbf{k}),
$$

where

$$
P_{i m}(\mathbf{k})=\delta_{i m}-k_{i} k_{m} k^{-2} .
$$

For simplicity, we follow Kraichnan ${ }^{15}$ and only consider the sharp Fourier cutoff filter,

$$
\mathcal{F}\left(k \mid k_{c}\right)=\left\{\begin{array}{ll}
1 & \text { for } k \leqslant k_{c} \\
0 & \text { for } k \geqslant k_{c}
\end{array} .\right.
$$

Because of this trivial analytical form, we need not distinguish the original and filtered velocity fields by special notation.

Filtering decomposes the energy transfer into the two parts

$$
T(k)=T^{<}\left(k \mid k_{c}\right)+T^{>}\left(k \mid k_{c}\right),
$$

where

$$
\begin{aligned}
T^{<}\left(k \mid k_{c}\right)= & \operatorname{Im}\left[P_{i m n}(\mathbf{k}) \int_{p, q \leqslant k_{c}} d \mathbf{p} d \mathbf{q} \delta(\mathbf{k}-\mathbf{p}-\mathbf{q})\right. \\
& \left.\times\left\langle u_{m}(\mathbf{p}, t) u_{n}(\mathbf{q}, t) u_{i}(-\mathbf{k}, t)\right\rangle\right]
\end{aligned}
$$

is the part of the total energy transfer due to interactions among resolved modes alone and

$$
T^{>}\left(k \mid k_{c}\right)=T(k)-T^{<}\left(k \mid k_{c}\right)
$$

represents the effect of unresolved interactions, in which either $p \geqslant k_{c}$ or $q \geqslant k_{c}$. In this setting, Kraichnan ${ }^{15}$ introduced the spectral eddy viscosity

$$
\nu_{t}\left(k \mid k_{c}\right)=-\frac{T^{>}\left(k \mid k_{c}\right)}{2 k^{2} Q(k)},
$$

so that the balance equation, Eq. (3), becomes

$$
2\left[\nu+\nu_{t}\left(k \mid k_{c}\right)\right] k^{2} Q(k)=F(k)+T^{<}\left(k \mid k_{c}\right) .
$$

This equation can be understood as a formal statement of the eddy viscosity assumption. It shows that an eddy viscosity must be scale- and cutoff-dependent.

To make the model determinate, a formula expressing $\nu_{t}$ in terms of the resolved field is required; in view of Eq. (10), this is equivalent to modeling the unresolved energy transfer $T^{>}$in terms of the resolved field. One standard model ${ }^{5,6}$ is

$$
\nu_{t}\left(k \mid k_{c}\right)=\nu_{t}^{+}\left(k \mid k_{c}\right) \sqrt{\frac{E\left(k_{c}\right)}{k_{c}}},
$$

where 


$$
\nu_{t}^{+}\left(k \mid k_{c}\right)=0.267+9.21 \exp \left(-3.03 k_{c} / k\right) .
$$

In applying Eq. (12), the quantity $E\left(k_{c}\right)$ is evaluated from the LES, not from the DNS. This model usually gives a satisfactory account of energy transfer in homogeneous turbulence. ${ }^{5}$ The question which we propose is whether this model also predicts turbulent time correlations well. This question will be discussed using comparisons with DNS and closure arguments.

\section{NUMERICAL CALCULATION OF TIME CORRELATIONS}

DNS and LES of isotropic homogeneous turbulence were performed using a standard pseudospectral Galerkin method. ${ }^{16}$ In DNS, the three-dimensional Navier-Stokes equations were solved on a cube of side $L_{B}=2 \pi$, with periodic boundary conditions in the three coordinate directions. The flow domain is discretized uniformly into $N^{3}$ grid points $(N=128)$, which defines the wave number components in Fourier space as $k_{j}= \pm n_{j}\left(2 \pi / L_{B}\right) \equiv \pm n_{j}$, where $n_{j}=0,1, \ldots$, $N / 2-1$ for $j=1,2,3$. Aliasing errors were removed by truncating the velocity field at higher wave numbers, $|k|>k_{\max }$ with $k_{\max }=N / 3$ (the two-thirds truncation method). Although relatively low resolution for computing spatial statistics, a $128^{3}$ simulation should give adequate representation of time correlations. ${ }^{17}$ The initial condition was an isotropic Gaussian field with energy spectrum

$$
E(k, 0) \propto\left(k / k_{0}\right)^{4} e^{-2\left(k / k_{0}\right)^{2}},
$$

where $k_{0}=4.68$ is the wave number at which the maximum of the energy spectrum occurs. A stationary turbulence was generated by maintaining constant total energy ${ }^{18}$ in each of the first two wave number shells $(0.5<k \leqslant 1.5$ and $1.5<k$ $\leqslant 2.5$ ), with the energy ratio between the two shells consistent with $k^{-5 / 3}$. The energy levels were 0.555440 and 0.159843 , respectively, for these first two wave number shells.

The spatial resolution of a spectral simulation is often monitored by the value of $k_{\max } \eta$, where $\eta \equiv\left(\nu^{3} / \epsilon\right)^{1 / 4}$ is the Kolmogorov microscale. This quantity should be greater than one for the smallest scales of the flow to be resolved. ${ }^{18}$ The value of $k_{\max } \eta$ was typically larger than 1.05 in our simulation.

The Fourier coefficients of the flow velocity were advanced in time using a second-order Adams-Bashforth method for the nonlinear term and an exact integration for the viscous term. ${ }^{16}$ The time step was chosen to ensure that the CFL number was 0.5 or less for numerical stability and accuracy. ${ }^{18}$

The resulting parameters of the DNS simulation are listed in Table I.

A mesh resolution of $64^{3}$ was used in the LES computations. Scales smaller than the grid spacing were modeled using the Chollet-Lesieur model (12), (13). The same forcing method used for DNS was applied to the first two wave number shells to maintain the energy in the resolved field of the LES. All other parameters in the LES are the same as in the DNS. The spectral codes for the DNS and LES were
TABLE I. DNS parameters and statistical quantities of the runs.

\begin{tabular}{ll}
\hline \hline Viscosity & $\nu=0.002$ \\
Average dissipation rate & $\epsilon=0.169$ \\
rms fluctuating velocity & $u^{\prime}=0.851$ \\
Integral length scale & $L_{f}=1.55$ \\
Taylor-scale Reynolds number & $R_{\lambda}=108.5$ \\
CFL number & 0.38 \\
Spatial resolution parameter & $k_{\max } \eta=1.05$ \\
Velocity derivative skewness & -0.47 \\
\hline \hline
\end{tabular}

developed and implemented on a Beowulf 96 CPU cluster computer at ICASE, NASA Langley Research Center, using the parallel fast-Fourier transformation algorithm. ${ }^{19}$ Figure 1 shows the energy spectra for the DNS and LES.

The correlation of Fourier amplitudes in the DNS field

$$
C(k, \tau)=\langle\mathbf{u}(\mathbf{k}, t+\tau) \cdot \mathbf{u}(-\mathbf{k}, t)\rangle
$$

is evaluated at a reference time $t$ after which the simulated flow becomes statistically stationary. The analogous quantity for the LES field is

$$
C\left(k \mid k_{c}, \tau\right)=\left\langle\mathbf{u}_{\mathrm{LES}}(\mathbf{k}, t+\tau) \cdot \mathbf{u}_{\mathrm{LES}}(-\mathbf{k}, t)\right\rangle .
$$

Subsequently, the time correlation for each wave number shell $|\mathbf{k}|=k$ is computed as a function of time lag $\tau$. The ensemble averaging is performed by averaging over the wave number shell $|\mathbf{k}|=k$. This procedure allows us to obtain smooth correlation functions.

\section{SIMULATION RESULTS}

The figures present the normalized time correlations

$$
\begin{aligned}
& c(k, \tau)=\frac{\langle\mathbf{u}(\mathbf{k}, t+\tau) \cdot \mathbf{u}(\mathbf{k}, t)\rangle}{\langle\mathbf{u}(\mathbf{k}, t) \mathbf{u}(-\mathbf{k}, t)\rangle}, \\
& c\left(k \mid k_{c}, \tau\right)=\frac{\left\langle\mathbf{u}_{\mathrm{LES}}(\mathbf{k}, t+\tau) \cdot \mathbf{u}_{\mathrm{LES}}(\mathbf{k}, t)\right\rangle}{\left\langle u_{\mathrm{LES}}(\mathbf{k}, t) \mathbf{u}_{\mathrm{LES}}(-\mathbf{k}, t)\right\rangle} .
\end{aligned}
$$

Figure 2 shows the time correlations of the DNS and LES fields for wave numbers $k=7,12,15,18$, spanning a range

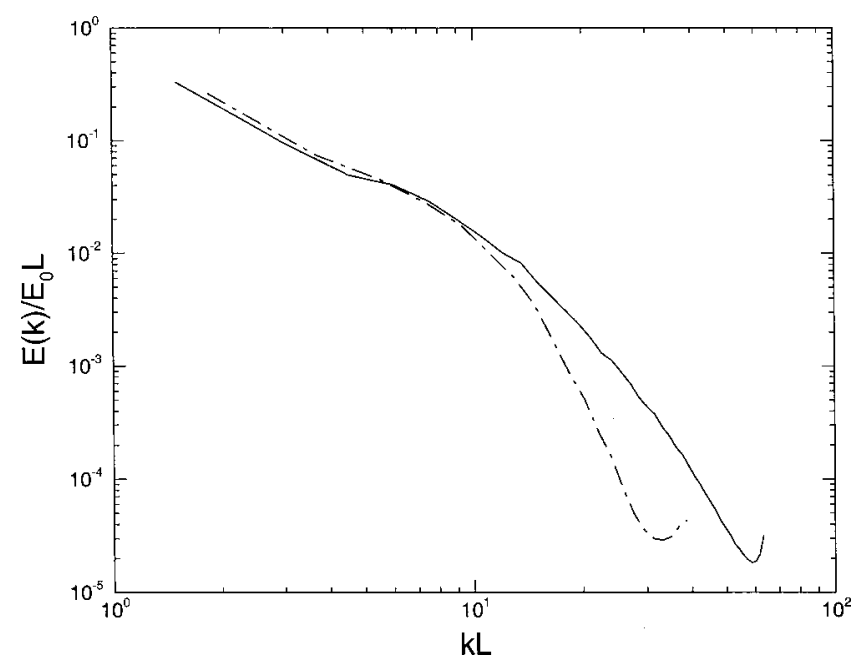

FIG. 1. Energy spectra for the DNS (solid line) and LES (dash-dotted line), where $L$ is the integral scale and $E_{0}$ total energy. 

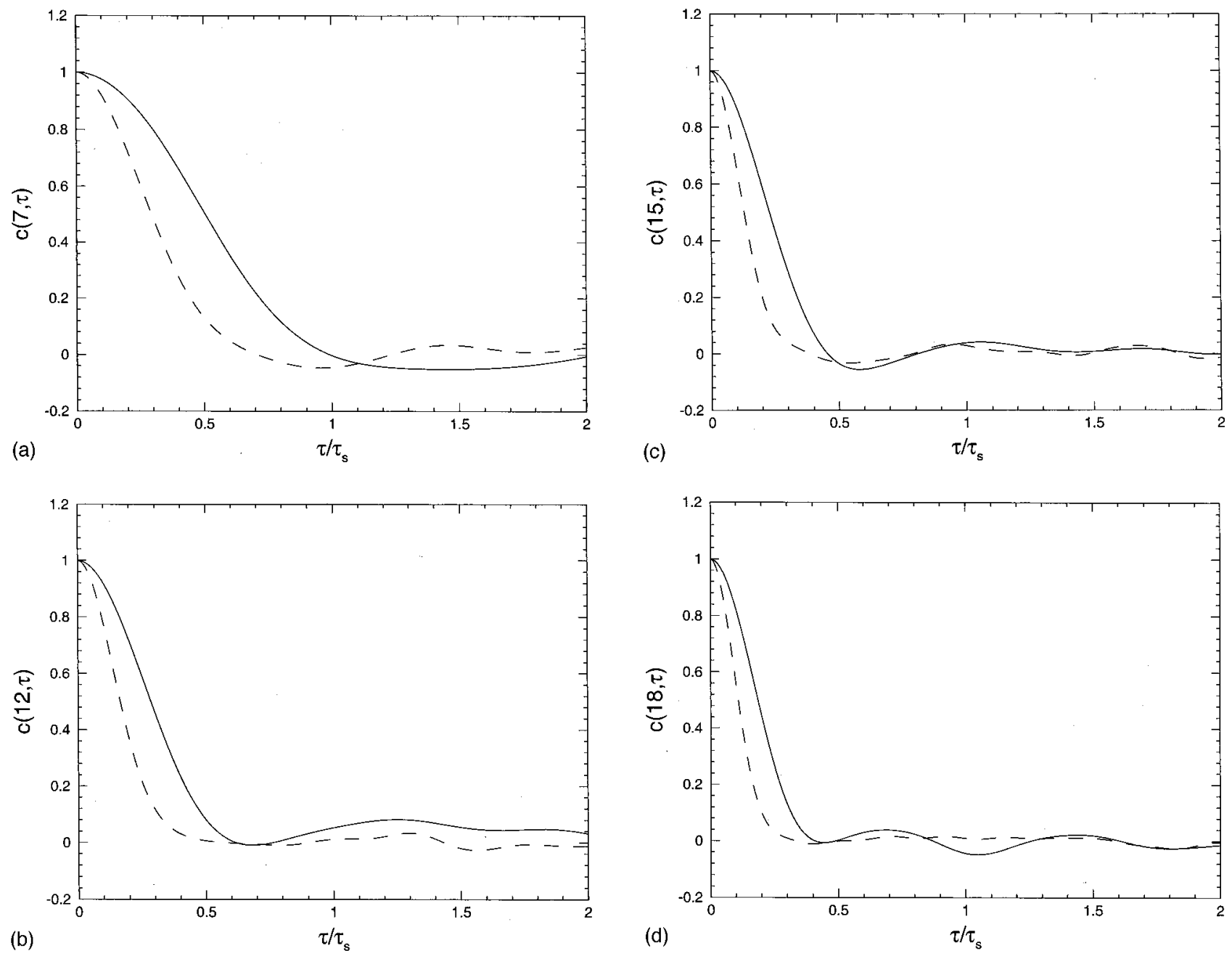

FIG. 2. Time correlation $c(k, \tau)$ vs time lag $\tau / \tau_{s}$. The solid line corresponding to the LES field and the dashed line to the DNS field: (a) $k=7$, (b) $k=12$, (c) $k=15$, (d) $k=18$.

of scales from the integral scale to the upper end of the resolved scale range. The time separation is normalized by $\tau_{s}=\left(u_{\mathrm{rms}} k_{\mathrm{min}}\right)^{-1}$ where $u_{\mathrm{rms}}$ is the rms fluctuating velocity and $k_{\min }=1$ is the largest scale in the system. This normalization is not essential to the numerical comparisons. Two conclusions are evident: first, both fields decorrelate more quickly at small scales than at large scales and second, the time correlations of the LES fields decay more slowly than those of the DNS fields.

In Fig. 3, these results are all plotted together, with the time axis defined by the scale-dependent similarity variable $\tau^{*}(k)=\tau u_{\mathrm{rms}} k$. This normalization causes excellent collapse of the correlation functions. The dynamic explanation has been discussed thoroughly by Kaneda. ${ }^{20}$ We note only that collapse on $\tau u_{\text {rms }} k$ is due to the fact that the Eulerian time correlations are dominated by the sweeping effect which is characterized by the value of $u_{\mathrm{rms}}$. This graph again makes evident the slower decorrelation of the LES field compared to the DNS field.

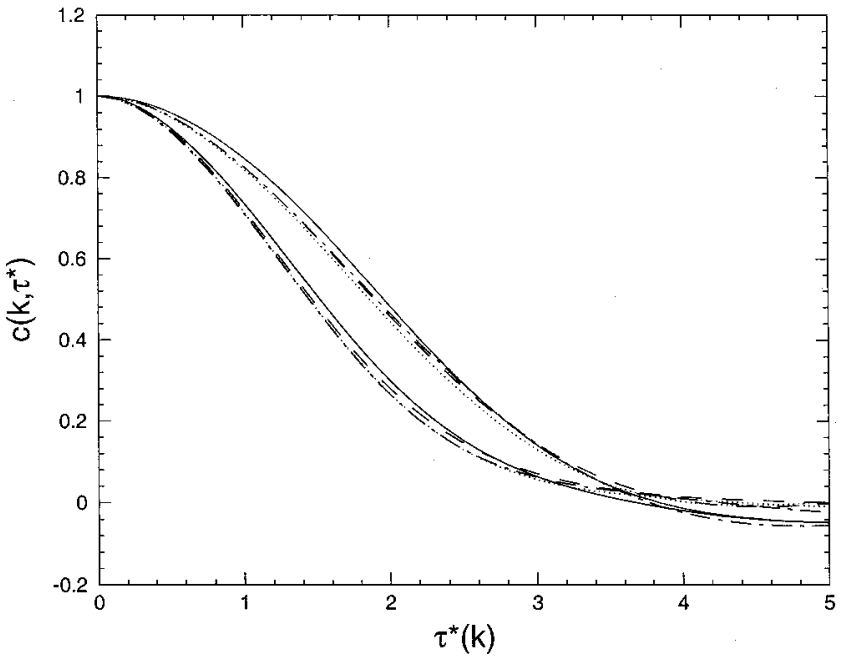

FIG. 3. Time correlation $c(k, \tau)$ vs time lag $\tau$ normalized by the sweeping time $u_{\mathrm{rms}} k$. The upper curves are from the LES and lower curves from the DNS. Other details are as in Fig. 1. 


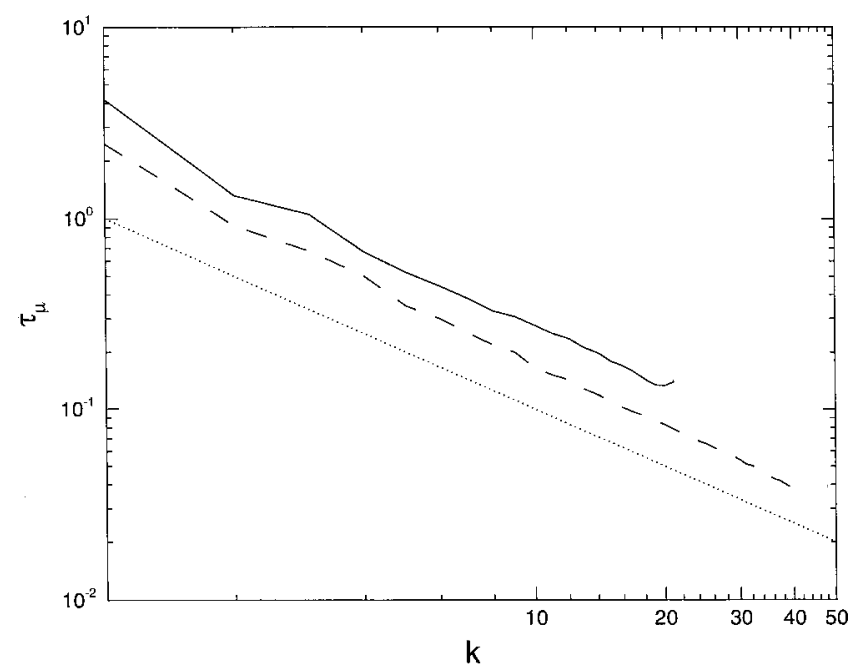

FIG. 4. Time microscales for the DNS and LES: solid line $\tau_{\mu}\left(k \mid k_{c}\right)$ (LES), dashed line $\tau_{\mu}(k)$ (DNS), and dotted line $k^{-1}$.

The time correlation function is often described by two scalar properties, the integral scale defined by

$$
\tau_{M}(k)=\int_{0}^{\infty} d \tau c(k, \tau)
$$

and the microscale defined by

$$
\tau_{\mu}(k)^{-2}=-\left.\frac{d^{2}}{d \tau^{2}} c(k, \tau)\right|_{\tau=0} .
$$

The integral scale $\tau_{M}(k)$ estimates the time required for the amplitude of a mode with wave number $k$ to become decorrelated with itself; the microscale is related to the zerocrossing rate of the time signal. The corresponding quantities for the LES field will be denoted by $\tau_{M}\left(k \mid k_{c}\right)$ and $\tau_{\mu}\left(k \mid k_{c}\right)$.

In Fig. 4, the microscales of the DNS and LES fields are compared over the range of wave numbers computed in the simulations. The plot shows that both microscales vary with wave number $k$ as $k^{-1}$, consistent with the sweeping hypothesis for Eulerian time correlations ${ }^{20,21}$ and with the collapse of the correlation functions shown in Fig. 2. The DNS and LES microscales are seen to be approximately proportional, with

$$
\frac{\tau_{\mu}\left(k \mid k_{c}\right)}{\tau_{\mu}(k)} \approx 1.8
$$

over the entire range of wave numbers considered. The LES microscale always exceeds the DNS microscale.

The same comparison for the integral scales is made in Fig. 5, which shows the ratio $\tau_{M}\left(k \mid k_{c}\right) / \tau_{M}(k)$ as a function of wave number $k$. Least-squares fitting of the data gives

$$
\frac{\tau_{M}\left(k \mid k_{c}\right)}{\tau_{M}(k)} \approx 1.6 .
$$

The LES integral scale also exceeds the DNS integral scale. Comparison between Eqs. (21) and (20) shows that the microscale is more sensitive to filtering than the integral scale.

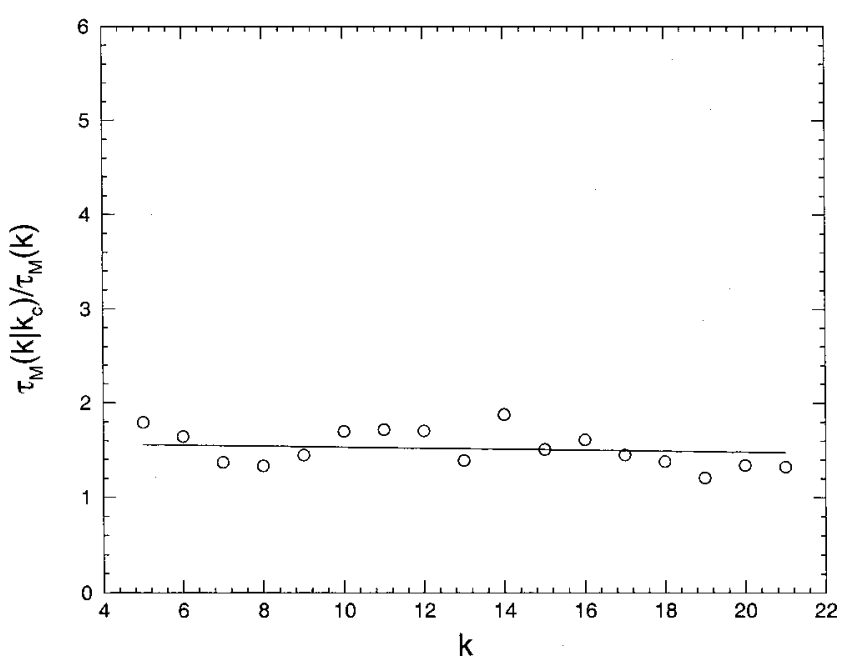

FIG. 5. The ratio of integral scales $\tau_{M}\left(k \mid k_{c}\right)$ (LES) and $\tau_{M}(k)$ (DNS) as a function of wave number $k$. The solid line is a least-squares fit.

\section{THE EFFECTS OF SUBGRID-SCALE MODELING ON TIME CORRELATION}

The Taylor expansion technique ${ }^{17,20}$ makes possible a simple analysis of the effects of eddy viscosity on the time microscale. To facilitate the analysis, we are assuming that initial DNS and LES velocity fields are identical and the question under investigation concerns the subsequent evolution under DNS and LES. The governing equations for the Navier-Stokes fields are

$$
\begin{aligned}
\left(\frac{\partial}{\partial t}+\nu k^{2}\right) u_{i}(\mathbf{k}, t)= & -\frac{i}{2} M_{i m n}(\mathbf{k}, \mathbf{p}, \mathbf{q}) u_{m}(\mathbf{p}, t) u_{n}(\mathbf{q}, t) \\
& +f_{i}(\mathbf{k}, t)
\end{aligned}
$$

where

$$
M_{i m n}(\mathbf{k}, \mathbf{p}, \mathbf{q})=P_{i m n}(\mathbf{k}) \int d \mathbf{p} d \mathbf{q} \delta(\mathbf{p}+\mathbf{q}-\mathbf{k})
$$

and $f_{i}(\mathbf{k}, t)$ is the large-scale random forcing which vanishes except at the forcing scales.

The governing equations for the LES fields are

$$
\begin{aligned}
\left(\frac{\partial}{\partial t}+\right. & {\left.\left[\nu+\nu_{t}\left(k \mid k_{c}\right)\right] k^{2}\right) u_{i}(\mathbf{k}, t) } \\
& =-\frac{i}{2} M_{i m n}^{<}(\mathbf{k}, \mathbf{p}, \mathbf{q}) u_{m}(\mathbf{p}, t) u_{n}(\mathbf{q}, t)+f_{i},
\end{aligned}
$$

where

$$
M_{i m n}^{<}(\mathbf{k}, \mathbf{p}, \mathbf{q})=P_{i m n}(\mathbf{k}) \int_{p, q \leqslant k_{c}} \delta(\mathbf{k}-\mathbf{p}-\mathbf{q}) d \mathbf{p} d \mathbf{q}
$$

restricts the nonlinear interaction to resolved modes alone.

The Taylor expansion of the time correlation (15) has the general form

$$
\begin{aligned}
& C(k, \tau)=C_{0}(k)+i C_{1}(k) \tau-C_{2}(k) \tau^{2} / 2+\cdots, \\
& C_{n}(k)=(-i)^{n}\left\langle\frac{\partial^{n} u(\mathbf{k}, t)}{\partial t^{n}} u(-\mathbf{k}, t)\right\rangle .
\end{aligned}
$$


The time derivatives of the Navier-Stokes fields can be calculated from Eq. (22). For negligible viscosity $\nu$ and wave numbers $k$ far from the forcing frequency, we obtain ${ }^{20}$

$$
\begin{aligned}
C_{0}(k)= & Q(k), \\
C_{1}(k)= & -\frac{i}{2} T(k), \\
C_{2}(k)= & \frac{1}{4} M_{i m n}(\mathbf{k}, \mathbf{p}, \mathbf{q}) M_{i r s}\left(\mathbf{k}, \mathbf{p}^{\prime}, \mathbf{q}^{\prime}\right) \\
& \times\left\langle u_{m}(\mathbf{p}) u_{n}(\mathbf{q}) u_{r}\left(\mathbf{p}^{\prime}\right) u_{s}\left(-\mathbf{q}^{\prime}\right)\right\rangle .
\end{aligned}
$$

Note that

$$
\tau_{\mu}(k)^{2}=\frac{C_{0}(k)}{C_{2}(k)} .
$$

In Eq. (29), the result for time stationary turbulence

$$
\langle\ddot{u}(k, t) u(k, t)\rangle=-\langle\dot{u}(k, t) \dot{u}(k, t)\rangle
$$

is applied.

In a steady state,

$$
\begin{aligned}
i C_{1}(k) & =\left\langle\frac{d u_{i}(\mathbf{k}, 0)}{d t} u_{i}(-\mathbf{k}, 0)\right\rangle \\
& =\frac{1}{2} \frac{d}{d t}\left\langle u_{i}(\mathbf{k}, 0) u_{i}(-\mathbf{k}, 0)\right\rangle \\
& =0 .
\end{aligned}
$$

In view of Eq. (28), this result is equivalent to $T(k)=0$ : a steady state implies zero net transfer into modes outside the production and dissipation ranges.

Similarly, the Taylor coefficients of time correlations in the LES velocity field, defined by analogy with Eq. (26) by

$$
C\left(k \mid k_{c}, \tau\right)=C_{0}\left(k \mid k_{c}\right)+i C_{1}\left(k \mid k_{c}\right) \tau-C_{2}\left(k \mid k_{c}\right) \tau^{2} / 2+\cdots
$$

can be obtained from Eq. (24) as

$$
\begin{aligned}
C_{0}\left(k \mid k_{c}\right)= & Q\left(k \mid k_{c}\right), \\
C_{1}\left(k \mid k_{c}\right)= & -\frac{i}{2}\left(T^{<}\left(k \mid k_{c}\right)-2 \nu_{t}\left(k \mid k_{c}\right) k^{2} Q\left(k \mid k_{c}\right)\right), \\
C_{2}\left(k \mid k_{c}\right)= & \frac{1}{4} M_{i m n}^{<}(\mathbf{k}, \mathbf{p}, \mathbf{q}) M_{i r s}^{<}\left(\mathbf{k}, \mathbf{p}^{\prime}, \mathbf{q}^{\prime}\right) \\
& \times\left\langle u_{m}(\mathbf{p}) u_{n}(\mathbf{q}) u_{r}\left(\mathbf{p}^{\prime}\right) u_{s}\left(\mathbf{q}^{\prime}\right)\right\rangle \\
& -\nu_{t}\left(k \mid k_{c}\right) k^{2} T^{<}\left(k \mid k_{c}\right) \\
& +\nu_{t}\left(k \mid k_{c}\right)^{2} k^{4} Q\left(k \mid k_{c}\right)
\end{aligned}
$$

In an ideal LES calculation, we would have

$$
Q\left(k \mid k_{c}\right)=Q(k) \text { for } k \leqslant k_{c} .
$$

Again,

$$
\tau_{\mu}\left(k \mid k_{c}\right)^{2}=\frac{Q(k)}{C_{2}\left(k \mid k_{c}\right)} .
$$

In the case of LES, the requirement that $C_{1}\left(k \mid k_{c}\right)=0$ in a steady state is equivalent to Kraichnan's definition of the spectral eddy viscosity, Eq. (10). Regardless of the definition of $v_{t}\left(k \mid k_{c}\right)$, energy transfer among the resolved modes adjusts itself so that $C_{1}\left(k \mid k_{c}\right)=0$.

Equation (36) can be simplified using the result $C_{1}\left(k \mid k_{c}\right)=0$ : in view of Eq. (35),

$$
C_{2}\left(k \mid k_{c}\right)=C_{2}^{<}\left(k \mid k_{c}\right)-\nu_{t}\left(k \mid k_{c}\right)^{2} k^{4} Q(k),
$$

where we have introduced the quantity

$$
\begin{aligned}
C_{2}^{<}\left(k \mid k_{c}\right) \equiv & \frac{1}{4} M_{i m n}^{<}(\mathbf{k}, \mathbf{p}, \mathbf{q}) M_{i r s}^{<}\left(\mathbf{k}, \mathbf{p}^{\prime}, \mathbf{q}^{\prime}\right) \\
& \times\left\langle u_{m}(\mathbf{p}) u_{n}(\mathbf{q}) u_{r}\left(\mathbf{p}^{\prime}\right) u_{s}\left(\mathbf{q}^{\prime}\right)\right\rangle,
\end{aligned}
$$

which represents the effect of interactions among resolved modes alone. Note that $v_{t}$ always decreases $C_{2}\left(k \mid k_{c}\right)$, hence it always increases the time microscale.

The quasinormality hypothesis results in

$$
\begin{aligned}
C_{2}^{<}\left(k \mid k_{c}\right)= & \frac{1}{2} M_{i m n}^{<}(\mathbf{k}, \mathbf{p}, \mathbf{q}) M_{i r s}^{<}\left(\mathbf{k}, \mathbf{p}^{\prime}, \mathbf{q}^{\prime}\right) \\
& \times P_{m r}(\mathbf{p}) P_{n s}(\mathbf{q}) Q(p) Q(q) .
\end{aligned}
$$

The geometric factor in Eq. (41) is positive, ${ }^{22}$ consequently

$$
C_{2}^{<}\left(k \mid k_{c}\right)<C_{2}(k) \text {. }
$$

Combining Eqs. (42) and (39),

$$
C_{2}\left(k \mid k_{c}\right)<C_{2}(k) \text {. }
$$

If the SGS model correctly predicts the energy levels of the resolved scales, we can divide Eq. (43) by $Q(k)$ and conclude that

$$
\tau_{\mu}\left(k \mid k_{c}\right)>\tau_{\mu}(k)
$$

as observed in the simulations. Thus, closure is consistent with the observation that the time microscale in the LES field is always larger than the microscale in the DNS field. The closure result, Eq. (41), also implies that $C_{2}\left(k \mid k_{c}\right)$ is an increasing function of $k_{c}$; as expected, increasing the resolution of the LES must improve the predicted microscale.

Because it is difficult to explore the effects of varying simulation parameters through DNS, it is useful to supplement the DNS with closure analysis. The most fundamental property of the integral defining $C_{2}(k)$ is that if it is evaluated on a cutoff Kolmogorov correlation function of the form

$$
Q(k)=\left\{\begin{array}{cc}
C_{\mathrm{K}} \epsilon^{2 / 3} k^{-5 / 3} /\left(2 \pi k^{2}\right), & k \geqslant k_{0} \\
Q_{0}(k), & k \leqslant k_{0}
\end{array},\right.
$$

where $Q_{0}(k)$ is an arbitrary function of $k$ which is bounded near $k=0$, then under the closure hypothesis of quasinormality, when $q \approx k_{0} \approx 0$ and consequently $\mathbf{p} \rightarrow \mathbf{k}$,

$$
C_{2}(k) \sim \epsilon^{4 / 3} k^{3} P_{i m n}(\mathbf{k}) P_{i r n}(\mathbf{k}) P_{m r}(\mathbf{k}) k_{0}^{-3 / 2} k^{-11 / 3}
$$

diverges for fixed $k$ as $k_{0} \rightarrow 0$. Note that

$$
c_{2}(k)=\frac{C_{2}(k)}{Q(k)} \sim \epsilon^{2 / 3}\left(k / k_{0}\right)^{2 / 3} k^{4 / 3}
$$

also diverges in this limit.

This is the well-known divergence property of Eulerian time correlations discovered by Kraichnan. ${ }^{23}$ It implies that if $k \gg k_{0}$, we can expect that the integrals defining both $C_{2}^{<}\left(k \mid k_{c}\right)$ and $C_{2}(k)$ will be dominated by the behavior of $Q$ 
TABLE II. The wave numbers $k_{0}, k$, and $k_{c}$.

\begin{tabular}{ccc}
\hline \hline$k_{0}$ & $k$ & $k_{c}$ \\
\hline $0.1,1.0,4.0$ & $8 \times 2^{-n}$ for $0 \leqslant n \leqslant 8$ & $16,32,64,128$ \\
\hline \hline
\end{tabular}

near the peak of the energy spectrum. If also $k \ll k_{c}$, then $C_{2}(k) \approx C_{2}^{<}\left(k \mid k_{c}\right)$. Thus, if $k_{0} \ll k \ll k_{c}, C_{2}(k)$ will be relatively unaffected by mode truncation, and should be well predicted by LES.

In a finite system in which $k_{0}$ is fixed, $C_{2}(k)$ is finite, because $Q_{0}(q)$ approaches zero when $q \rightarrow 0$. For fixed $k_{0}$, if $k \approx k_{0}$, then Eq. (46) shows that the contribution to $C_{2}(k)$ from scales $q \approx 0$ is of order $k_{0}^{2 / 3}$. Similarly, Eq. (47) shows that the contribution to $c_{2}(k)$ from these scales is of order $k_{0}^{4 / 3}$. Thus, $C_{2}(k)$ and $c_{2}(k)$ are no longer dominated by contributions from small $q$; instead, these contributions are small, and the effect of the cutoff at $k_{c}$ can be stronger.

The conclusion of these two arguments is that in high Reynolds number turbulence with $k_{0} \ll k_{d}$, where $k_{d}$ is the inverse Kolmogorov scale, the time microscale can be well predicted by LES for scales $k$ satisfying $k_{0} \ll k \ll k_{c}<k_{d}$, but the effect of mode truncation can be stronger if $k \approx k_{0}$ or if $k \approx k_{c}$, whether the Reynolds number is large or not. In particular, in low Reynolds number turbulence in which $k_{0}$ $\approx k_{d}$, the effect of the cutoff can always be significant.

To quantify the effect of proximity to the cutoff scales on time correlations, we evaluated the integral of Eq. (41) numerically for the von Kármán spectrum

$$
Q(k)=C \epsilon^{2 / 3} k_{0}^{-5 / 3}\left(k / k_{0}\right)^{4}\left[1+\left(k / k_{0}\right)^{2}\right]^{-17 / 6} k^{-2},
$$

where $k_{0}$ defines the peak of the energy spectrum. In this integration, the wave vectors are restricted by the conditions $p, q<k_{c}$. The problem has two parameters $k_{0}, k_{c}$; these cases are considered in Table II.

The results are given in Table III.

The results shown in Table III agree with the abovegiven arguments: the cutoff effects are largest when $k_{0}$ is largest and increase as $k$ approaches $k_{0}$; we also note that the time microscale for scales $k<k_{0}$ below the integral scale are even more strongly affected by the cutoff. The results in Table III do not include the effect of eddy viscosity: for the

TABLE III. The effects of proximity to the cutoff scales on time correlations.

\begin{tabular}{cccc}
\hline \hline$k / k_{0}$ & $C_{2}(k \mid 32) / C_{2}(k \mid 16)$ & $C_{2}(k \mid 64) / C_{2}(k \mid 32)$ & $C_{2}(k \mid 128) / C_{2}(k \mid 64)$ \\
\hline & & $k_{0}=4.0$ & \\
2.000 & 1.107 & 1.062 & 1.037 \\
0.500 & 1.137 & 1.073 & 1.041 \\
0.125 & 1.146 & 1.075 & 1.042 \\
& & $k_{0}=1.0$ & \\
2.000 & 1.037 & 1.022 & 1.013 \\
0.500 & 1.041 & 1.024 & 1.014 \\
0.125 & 1.042 & 1.025 & 1.016 \\
& & $k_{0}=0.1$ & 1.002 \\
2.5000 & 1.007 & 1.004 & 1.003 \\
1.2500 & 1.007 & 1.004 & \\
\hline \hline
\end{tabular}

Chollet-Lesieur model-Eq. (12) - this effect, which is proportional to the energy spectrum at the cutoff wave number, is small and is consequently not shown.

None of the ratios in Table III is close to the value $c_{2}(k) / c_{2}\left(k \mid k_{c}\right) \approx 1.8$ found in our DNS. The reason is that the von Kármán spectrum happens not to apply well to either the DNS or LES spectrum. Modeling these spectra by

$$
\begin{aligned}
& E_{\mathrm{DNS}}(k)=\left\{\begin{array}{cc}
0 & \text { for } k \leqslant 1.0 \\
0.7 k^{-1.67} & \text { for } 1.0 \leqslant k \leqslant 15.3, \\
700 k^{-4.20} & \text { for } 15.3 \leqslant k \leqslant 60.0
\end{array}\right. \\
& E_{\mathrm{LES}}(k)=\left\{\begin{array}{cc}
0 & \text { for } k \leqslant 1.0 \\
0.7 k^{-1.67} & \text { for } 1.0 \leqslant k \leqslant 11.5 \\
5000 k^{-5.30} & \text { for } 11.5 \leqslant k \leqslant 30.0
\end{array}\right.
\end{aligned}
$$

numerical evaluation of the ratio $c_{2}(k) / c_{2}\left(k \mid k_{c}\right)$ gave values in the range 1.6-2.0.

The disparity in the ratios $c_{2}(k) / c_{2}\left(k \mid k_{c}\right) \approx 1.0-1.15$ found in Table III and $c_{2}(k) / c_{2}\left(k \mid k_{c}\right) \approx 1.8$ for the numerical simulations is surprising. However, we should consider that Table III was constructed by making the idealization that the LES spectrum is simply the cutoff DNS spectrum as in Eq. (37). It is therefore likely that the ratios in Table III are unrepresentatively low. The numerical simulations have applied the Chollet-Lesieur spectral eddy viscosity, which is derived theoretically for high Reynolds number turbulence, in a relatively low Reynolds number simulation. The LES is probably far from optimal. It is therefore also likely that the ratios from the simulations are unrepresentatively high. In any case, our goal is not to predict this ratio, which depends on the flow and the SGS model, but to show that it can be significantly larger than one.

\section{DISCUSSION}

In most previous applications, LES has been judged by its ability to predict single-time flow properties like the mean velocity profile, turbulent kinetic energy, and Reynolds stresses. SGS model development has therefore been focused on improving the predictions of these quantities. The time statistics of the LES do not enter such comparisons directly. The recent increased attention given to unsteady problems in aerodynamics suggests that LES will be needed for timeaccurate calculations in the future; even if the time accuracy required is only statistical, this emphasis will impose new requirements on SGS modeling.

A relatively new application of LES to unsteady flow is the computation of turbulent sound sources. In this problem, the Lighthill acoustic analogy ${ }^{24}$ shows that the sound source depends on the space-time properties of turbulence. Since unsteady information is required, LES is a natural approach; however, investigation of the accuracy of LES in this application has begun only recently.

In a recent assessment of LES-based sound radiation calculations, Seror et al. ${ }^{25}$ found that SGS modeling suppresses the sound radiated by the unresolved scales. It is not difficult to use turbulence theory to model the subgrid sound 
sources $^{26,27}$ without reformulating the SGS model; however, the possible modification of the frequency distribution presents a potentially more difficult problem.

The problem thereby arises of constructing SGS models which are faithful both to the energetics of turbulence and to its time correlation properties. One way to model the decorrelating effects of the unresolved scales is by adding a random force to the SGS model. The introduction of such Langevin models as SGS models has been suggested by Bertoglio, ${ }^{12}$ Leith, ${ }^{13}$ and Chasnov ${ }^{14}$ in connection with the predictability problem for turbulence. The effect of an added random force is easily understood in the present context: if the large-scale forcing $f_{i}$ in Eq. (24) is replaced by a random force which acts at all scales, then a simple calculation shows that Eq. (39) is replaced by

$$
\begin{aligned}
C_{2}\left(k \mid k_{c}\right)= & C_{2}^{<}\left(k \mid k_{c}\right)+M_{i m n}^{<}(\mathbf{k})\left\langle u_{m}(\mathbf{p}) u_{n}(\mathbf{q}) f_{i}(-\mathbf{k})\right\rangle \\
& -\nu_{t}\left(k \mid k_{c}\right)^{2} k^{4} Q(k)+\left\langle f_{i}(\mathbf{k}) f_{i}(-\mathbf{k})\right\rangle .
\end{aligned}
$$

Since the random force increases the inverse microscale, it is possible that the force correlation could be adjusted to compensate for the effect of filtering and SGS modeling.

\section{CONCLUSIONS}

Numerical simulation and closure arguments suggest that SGS modeling generates LES fields which are more correlated than the corresponding DNS fields. This can be true even if the SGS model predicts single-time properties well. The increased time correlation cannot be attributed to filtering alone; indeed, filtering alone does not change the time correlations of velocity Fourier modes. Instead, by modifying the phase relations among modes, SGS modeling alters their correlation properties. This is reflected in increased temporal correlations.

The present paper considers only Eulerian time correlations, which are relevant to problems involving wave radiation and scattering. In very high Reynolds number turbulent flows, the long wavelength divergence of Eulerian time correlations implies that the time correlations of scales far from both the integral scale and the subgrid cutoff should be well predicted by LES. Increased correlation will be significant in moderate Reynolds number turbulence and in high Reynolds number LES for scales close to the integral scale or the filter scale.

A representative application of this effect is in sound radiation calculations where, by increasing the turbulent time scales, it could cause a shift in the computed radiated sound lower frequencies. Adding a random force to the SGS model is a possible route to improving the time correlation predictions.

\section{ACKNOWLEDGMENTS}

The authors are very grateful to Y. Kaneda for many helpful discussions. They also thank T. Gotoh for a discus- sion of ensemble averaging of Fourier amplitudes. G.-W.H. acknowledges the support of the program "Nonlinear Science."

${ }^{1}$ J. Smagorinsky, "General circulation experiments with the primitive equations. I. The basic experiment," Mon. Weather Rev. 91, 99 (1963).

${ }^{2}$ M. Germano, U. Piomelli, P. Moin, and W. H. Cabot, "A dynamic subgridscale eddy viscosity model," Phys. Fluids A 3, 1760 (1991).

${ }^{3}$ J. Bardina, J. H. Ferziger, and W. C. Reynolds, "Improved subgrid scale models for large-eddy simulation," AIAA Pap. 80-1357.

${ }^{4} \mathrm{~K}$. Horiuti, "A new dynamic two-parameter mixed model for large-eddy simulation," Phys. Fluids 9, 3443 (1997).

${ }^{5}$ J.-P. Chollet and M. Lesieur, "Parameterization of small scales of threedimensional isotropic turbulence utilizing spectral closure," J. Atmos. Sci. 38, 2747 (1981).

${ }^{6} \mathrm{O}$. Métais and M. Lesieur, "Spectral large-eddy simulation of isotropic and stably stratified turbulence," J. Fluid Mech. 239, 157 (1992).

${ }^{7}$ R. Rubinstein and Y. Zhou, "The frequency spectrum of sound radiated by isotropic turbulence," Phys. Lett. A 267, 379 (2000).

${ }^{8}$ U. Piomelli, C. L. Streett, and S. Sarkar, "On the computation of sound by large-eddy simulation,” J. Eng. Math. 32, 217 (1997).

${ }^{9}$ C. Seror, P. Sagaut, C. Bailly, and D. Juvé, "Subgrid scale contribution to noise production in decaying isotropic turbulence," AIAA J. 38, 1795 (2000).

${ }^{10}$ C. E. Leith and R. H. Kraichnan, "Predictability of turbulent flows," J. Atmos. Sci. 29, 1041 (1972).

${ }^{11}$ G. L. Eyink, "Fluctuation-response relations for multi-time correlations," Phys. Rev. E 62, 210 (2000).

${ }^{12}$ J.-P. Bertoglio, "A stochastic subgrid model for sheared turbulence," Lect. Notes Phys. 230, 100 (1984).

${ }^{13}$ C. E. Leith, "Stochastic backscatter in a subgrid-scale model: Plane shear mixing layer," Phys. Fluids A 2, 297 (1990).

${ }^{14}$ J. R. Chasnov, "Simulation of the Kolmogorov inertial subrange using an improved subgrid model," Phys. Fluids A 3, 188 (1991).

${ }^{15}$ R. H. Kraichnan, "Eddy viscosity in two and three dimensions," J. Atmos. Sci. 33, 1521 (1976).

${ }^{16} \mathrm{~A}$. Vincent and M. Meneguzzi, "The spatial structure and statistical properties of homogeneous turbulence," J. Fluid Mech. 225, 1 (1991).

${ }^{17}$ Y. Kaneda, "Taylor expansion in powers of time of Lagrangian and Eulerian two-point two time correlation in turbulence," Phys. Fluids 11, 2154 (1999).

${ }^{18}$ V. Eswaran and S. B. Pope, "An examination of forcing in direct numerical simulations of turbulence," Comput. Fluids 16, 257 (1988).

${ }^{19}$ P. Dmitrul, L. P. Wang, W. H. Matthaeus, R. Zhang, and D. Seckel, "Scalable parallel FFT for spectral simulations on a Beowulf cluster," Parallel Comput. 27, 1921 (2001)

${ }^{20}$ Y. Kaneda, "Lagrangian and Eulerian time correlations in turbulence," Phys. Fluids A 5, 2835 (1993).

${ }^{21}$ R. H. Kraichnan, "Kolmogorov's hypotheses and Eulerian turbulence theory," Phys. Fluids 7, 1723 (1964).

${ }^{22}$ S. A. Orszag, "Lectures on the statistical theory of turbulence," in Fluid Mechanics, edited by J. Balian and J.-P. Preuve (Gordon and Breach, New York, 1973).

${ }^{23}$ R. H. Kraichnan, "Structure of isotropic turbulence at very high Reynolds numbers," J. Fluid Mech. 5, 497 (1959).

${ }^{24}$ M. J. Lighthill, "On sound generated aerodynamically. I. General theory," Proc. R. Soc. London, Ser. A 211, 1107 (1952).

${ }^{25}$ C. Seror, P. Sagaut, C. Bailly, and D. Juvé, "On the radiated noise computed by large-eddy simulation," Phys. Fluids 13, 476 (2001).

${ }^{26}$ C. Bailly, P. Lafon, and S. Candel, "Subsonic and supersonic jet noise prediction from statistical source models," AIAA J. 35, 1688 (1997).

${ }^{27}$ R. Rubinstein and Y. Zhou, "Characterization of sound radiation by unresolved scales of motion in computational aeroacoustics," ICASE Report No. 99-39, 1999. 